\title{
Best First Line Tyrosine Kinase Inhibitor for Chronic Phase Chronic Myeloid Leukemia in Adults: A Network Meta-Analysis
}

Claudia Vener ( $\square$ claudia.vener@unimi.it )

University of Milan

Bruno Fattizzo

La Fondazione IRCCS Ca\' Granda: Ospedale Maggiore Policlinico

Federico Ambrogi

University of Milan

Giuseppe Saglio

University of Study of Turin

Gabriella Pravettoni

University of Milan

Doris Kopp-Heim

University of Bern

Cinzia Del Giovane

University of Bern

\section{Protocol}

Keywords: CML, TKI, imatinib, dasatinib, nilotinib, bosutinib, ponatinib

Posted Date: November 5th, 2020

DOl: https://doi.org/10.21203/rs.3.rs-102006/v1

License: (9) This work is licensed under a Creative Commons Attribution 4.0 International License.

Read Full License 


\section{Abstract}

\section{Background}

For many years chronic myeloid leukemia (CML) remained a leukemic subtype in which little improvement was gained with regard to overall survival (OS). Imatinib, the first tyrosine kinase inhibitor (TKI), completely changed patients' life expectancy. Although many studies have compared the use of imatinib and more recent TKIs in patients with newly diagnosed chronic phase (CP) CML, they have not established which is the most effective. Since there are no randomised controlled trials (RCTs) that compare head-to-head second- and third-generation TKIs, a network meta-analysis (NMA) is required to identify the best TKI to be preferably used.

Methods

This systematic review and NMA of RCTs will compare the efficacy and safety of imatinib vs secondgeneration (dasatinib, nilotinib, bosutinib) and third-generation TKIs (ponatinib) in adults with newly diagnosed Philadelphia Chromosome-Positive $(\mathrm{Ph}+) \mathrm{CP} C \mathrm{CM}$, concentrating on OS, progression-free survival (PFS), and hematological and non-hematological adverse events (AEs). The databases PubMed, EMBASE and Cochrane CENTRAL will be systematically searched, as well as other sources to identify published and unpublished studies. The risk of bias (RoB) of the included studies will be assessed using the Cochrane risk of bias tool; moreover, the confidence in network meta-analysis estimates will be evaluated with the CINeMA tool.

\section{Discussion}

The results of this systematic review and NMA are expected to suggest a specific TKI to be used for patients with newly CP CML. It remains valid that the choice of the optimal drug should consider AEs and co-morbidities as well as molecular/cytogenetic responses and transformation rates.

Systematic review registration

The review protocol PROSPERO registration number is: CRD42020190444. This protocol is written in accordance with the guidelines of Preferred Reporting Items for Systematic Review and Meta-Analysis Protocols (PRISMA-P). The PRISMA-P checklist can be found in Additional file 1.

\section{Key Points}

- Selecting the best first-line tyrosine kinase inhibitor (TKI) for patients with chronic phase (CP) chronic myeloid leukemia (CML) on the basis of efficacy and toxicity is of paramount importance.

- The protocol includes systematic review; network meta-analysis; Cochrane risk of bias (RoB); confidence in network meta-analysis (CINeMA). 


\section{Background}

For many years, chronic myeloid leukemia $(\mathrm{CML})$ remained a leukemic subtype in which little improvement was gained with regard to overall survival (OS). ${ }^{1-3}$

Imatinib (Glivec, Novartis), the first tyrosine kinase inhibitor (TKI), completely changed patients' life expectancy. ${ }^{4}$ It was approved in 2001 (in Europe and the USA) for all CML phases and, as its patent has expired, is now available as a generic drug. ${ }^{5}$

Two second-generation TKIs, dasatinib (Sprycel, Bristol-Myers Squibb) and nilotinib (Tasigna, Novartis), were approved in the USA and Europe in 2006-2007 as second-line treatment for patients resistant to, or intolerant of previous treatment (including imatinib): dasatinib in all CML phases, and nilotinib only in the chronic phase (CP) or accelerated phase (AP). ${ }^{6-9}$ Since 2010-2011, both have been authorised for the firstline treatment of newly diagnosed Philadelphia chromosome-positive ( $\mathrm{Ph}+)$ adult $\mathrm{CP} C \mathrm{CML} .{ }^{10,11}$ Another second-generation TKI, bosutinib (Bosulif, Pfizer), was licensed in the USA in 2012 and in Europe in 2013 for the treatment of adults with $\mathrm{CP}, \mathrm{AP}$ or blast phase (BP) Ph+ CML who are resistant to, or intolerant of previous treatment with one or more TKIs ${ }^{12-14}$ and, in December 2017, the indication was extended in the USA to include newly diagnosed adult Ph+ CP CML.

The third-generation TKI, ponatinib (Iclusig, ARIAD), was approved in the USA in 2012 and in Europe in 2013 for the treatment of adults with $\mathrm{CP}, \mathrm{AP}$ or $\mathrm{BP} \mathrm{Ph}+\mathrm{CML}$ who are resistant to, or intolerant of other TKIs, and those with $\mathrm{CP}, \mathrm{AP}$ or $\mathrm{BP} \mathrm{Ph}+\mathrm{CML}$ and the T315I mutation known to be involved in resistance to imatinib. ${ }^{15,16}$

Although many studies have compared the use of imatinib and more recent TKIs in patients with newly diagnosed Ph+ CP CML, they have not established which is the most effective because they have not always considered the same outcomes and often evaluated them at different times; only some studies provided complete OS and progression-free survival (PFS) data; and the large number of adverse events (AE) are not specific to a single TKI. ${ }^{17-20}$ Furthermore, the 2017 European Society for Medical Oncology (ESMO) and 2018 National Comprehensive Cancer Network (NCCN) CML guidelines did not make any precise recommendations that would help clinicians decide. ${ }^{21-22}$

Otherwise, our previous systematic review and meta-analysis of randomised controlled trials (RCTs) ("First-line imatinib vs second- and third-generation TKIs for chronic phase CML: a systematic review and meta-analysis" by C. Vener et al., PROSPERO Registration No. CRD42016032903) provided complete, updated and precise comparative information concerning the use of TKIs in patients with newly diagnosed adult CP CML in terms of OS and PFS at various time points, clinical and biological response variables, and the most relevant hematological and non-hematological AEs. ${ }^{23}$ Biases and the quality of the evidence were assessed using the Cochrane risk of bias tool (RoB) and the Grading of Recommendations Assessment, Development and Evaluation (GRADE) method. ${ }^{24-25}$ On the basis of secondary efficacy outcomes, we suggested that patients with newly diagnosed CP CML without co- 
morbidities should receive second- or third-generation TKIs; however, on the basis of toxicity outcomes, patients with co-morbidities should preferably be treated with imatinib. Unfortunately, we couldn't recommend a specific newer TKI because there are no head-to-head RCTs between second- and thirdgeneration TKIs, but we could only compare imatinib with newer TKIs considered as a whole. Network meta-analysis (NMA) is a statistical method that allows researchers to compare (indirectly) treatments that have never been compared head-to-head in a trial. ${ }^{26}$ For our project, a NMA is therefore required to identify the TKI with the best efficacy-safety profile to be preferably used.

\section{Methods/design}

\section{Review question}

This systematic review and NMA of RCTs will compare the efficacy and safety of imatinib vs secondgeneration (dasatinib, nilotinib, bosutinib) and third-generation TKIs (ponatinib), considered individually, in adults with newly diagnosed Ph+ CP CML. The evaluated outcomes will be OS, PFS, response, and safety (hematological and non-hematological AEs) (review protocol PROSPERO registration number: CRD42020190444). ${ }^{27}$ This protocol is written in accordance with the guidelines of Preferred Reporting Items for Systematic Review and Meta-Analysis Protocols (PRISMA-P). The PRISMA-P checklist can be found in Additional file 1.

\section{Searches}

To identify the published or unpublished studies of interest we will systematically search for RCTs or quasi-RCTs in the databases PubMed, EMBASE, the Cochrane Central Register of Controlled Trials (CENTRAL) and ClinicalTrials.gov, from 1990 to 2020. Our search stategy is reported in Additional file 2 (Appendix). We will scan the reference lists of the articles identified, recent systematic reviews, metaanalyses and guidelines to identify other pertinent studies. We will also screen the abstracts of the main conferences in the field (the American Society of Hematology, ASH; the American Society of Clinical Oncology, ASCO; the European Haematology Association, EHA; the Italian Society of Hematology, SIE). We will invite all of the manufacturers of the TKIs in question to provide any unpublished material. No language restrictions will be imposed.

\section{Participants/population}

Adult patient ( $>=18$ years old) with newly diagnosed (within 6 months) $\mathrm{Ph}+\mathrm{CP} C \mathrm{CM}$, by peripheral blood or bone marrow (BM) cytogenetics or fluorescent in situ hybridization (FISH), or the detection of Bcr-Abl transcript by reverse transcription polymerase chain reaction (RT-PCR); treatment with a TKI prior to study entry is not allowed except for no more than 2 weeks in duration of imatinib; any other medical treatment for CML prior to study entry for longer than 2 weeks is not allowed with the exception of hydroxyurea and/or anagrelide. 


\section{Intervention}

Imatinib, at any dose.

\section{Comparator}

Second generation TKIs, dasatinib, nilotinib, bosutinib, at any dose; third generation TKI, ponatinib, at any dose. We will exclude studies with interventions other than TKI, e.g. interferon-a, chemotherapy, stem cell transplantation, or best supportive care.

\section{Types of study to be included initially}

Published and unpublished RCTs. Trials for which it was unclear whether the method of randomisation provided adequate allocation concealment (quasi-RCTs) and open-label RCTs will be considered, but their quality will be taken into account. We will exclude non-randomised studies.

\section{Primary outcome}

Primary efficacy: 1) PFS; 2) OS.

\section{Secondary outcome}

\section{Secondary Efficacy:}

1) Complete cytogenetic response (CCyR), at and by 12 months of therapy. CCyR is defined as the absence of $\mathrm{Ph}+$ metaphases, determined on the basis of $\mathrm{G}$-banding in at least 20 cells in metaphase per BM sample.

2) Early molecular response (EMR, BCR-ABLIS $\leq 10 \%$ ), at 3 months of therapy.

3) Major molecular response (MMR, BCR-ABLIS $\leq 0.1 \%$ ), at 3 and at 12 months of therapy.

4) $M R 4$ (BCR-ABLIS $\leq 0.01 \%$ ) and MR4.5 (BCR-ABLIS $\leq 0.0032 \%)$, at any time of therapy.

5) Transformations to both AP and BP CML (European LeukemiaNet 2006 criteria; not included clonal evolution), during study treatment.

6) Discontinued treatment, at any time of therapy.

\section{Primary safety:}

Grade 3-4 hematologic toxicities (anemia, neutropenia, thrombocytopenia).

\section{Secondary safety:}


Grade 3-4 non-hematologic toxicities: cardiovascular events, pancreatic effects, hepatic effects, cutaneous effects, gastrointestinal effects, fluid retention (pleural and pericardial effusion), musculoskeletal disorders, infectious events, QT prolongation.

\section{Data extraction}

The full text of the potentially eligible studies will be retrieved and independently assessed for eligibility by two review team members. The same two review authors will independently extract the following information from each study: publication date, study design characteristics, and data on the interventions and control arms. Any disagreement between them over the eligibility of particular studies will be resolved through discussion with a third reviewer. For data not showed, relevant to the NMA, will be requested the authors to provide them.

\section{Risk of bias (quality) assessment}

Two review authors will independently assess the RoB of the included studies using the Cochrane risk of bias tool. ${ }^{24}$ We will consider the following domains: selection bias (sequence generation, allocation concealment), detection bias (blinding of outcome assessors), performance bias (blinding of participants and personnel), attrition bias (incomplete outcome data), reporting bias. In addition, we will assess the confidence in network meta-analysis estimates by using the new CINeMA tool. ${ }^{28}$

\section{Strategy for data synthesis}

We will provide a narrative synthesis of the findings from the included studies, structured around the type of intervention, target population characteristics, type of outcome and intervention content.

We will estimate treatment effects from each study by calculating risk ratios (for dichotomous outcomes) and hazard ratios (for OS and PFS) and relative $95 \%$ confidence intervals. We will perform standard pairwise meta-analysis and NMA in a frequentist context with a random effect model. We will present results from pairwise meta-analysis and NMA as summary relative effect sizes.

Within each pairwise comparison in standard pairwise meta-analysis, we will assess clinical heterogeneity comparing the data on potential effect modifiers and the presence of statistical heterogeneity by visual inspection of the forest plots and by calculating the $\mathrm{I}^{2}$ statistic. ${ }^{29}$ An I-squared value smaller than $50 \%$ reveals low heterogeneity, I-squared included between 50 and $75 \%$ moderate heterogeneity, and I-squared greater than $75 \%$ substantial heterogeneity.

Within NMA, we will assume a common estimate for the heterogeneity variance. We will compare the distribution of potential effect modifiers across different pairwise comparisons to assess transitivity across treatment comparisons. We will assess the presence of statistical heterogeneity in the entire network considering the magnitude of the common heterogeneity parameter. We will also evaluate the statistical disagreements between direct and indirect effect sizes, called incoherence, by using local and 
global approaches. Locally, we will use the loop-specific approach ${ }^{30}$; we will use the 'design-by-treatment' Q-statistic in the entire network. ${ }^{31}$

We will determine a treatment hierarchy by using the surface under the cumulative ranking curve (SUCRA) and mean ranks. ${ }^{32}$ All analyses will be conducted in Stata v. 12.0 statistical software.

\section{Analysis of subgroups or subsets}

We will perform a sensitivity analysis considering only low RoB studies. ${ }^{24}$

\section{Dissemination plans}

A scientific paper will be submitted to a leading journal in this field.

\section{Details of any existing review of the same topic by the same authors}

"First-line imatinib vs second- and third-generation TKIs for chronic phase CML: a systematic review and meta-analysis" by C. Vener et al., PROSPERO Registration No. CRD42016032903. ${ }^{23}$

\section{Discussion}

Although many studies have compared the use of imatinib and more recent TKIs in patients with newly diagnosed $\mathrm{Ph}+\mathrm{CP} C \mathrm{CML}$, they have not established which is the most efficacious. ${ }^{6-9,13,14,16}$ Furthermore, the 2017 ESMO and 2018 NCCN CML guidelines do not make precise recommendations that would help clinicians decide. ${ }^{21,22}$

Our previous systematic review and meta-analysis of RCTs provides complete, updated and precise comparative information concerning the use of TKIs in patients with newly diagnosed adult CP CML in terms of OS and PFS at various time points, clinical and biological response variables, and the most relevant hematological and non-hematological AEs. ${ }^{23}$ Biases and the quality of the evidence were assessed using the Cochrane risk of bias tool and the GRADE method, ${ }^{24-25}$ and the two databases created are available for data sharing purposes.

Unfortunately, we could not recommend a specific newer TKI because there are no head-to-head RCTs between second- and third-generation TKIs and concluded that a NMA is required. NMA is more powerful method than conventional pairwise meta-analysis because NMA can simultaneously compare multiple treatments, even in absence of head-to-head trials, and rank them according to their efficacy and safety. ${ }^{33}$ It should however be noted, that besides cumulative data on OS, PFS, and safety, the choice of the optimal TKI for patients with newly CP CML should always consider AEs and co-morbidities as well as molecular/cytogenetic responses and transformation rates.

\section{Abbreviations}


AE: Adverse Event; AP: Accelerated Phase; ASCO: American Society of Clinical Oncology; ASH: American Society of Hematology; Bcr-Abl: Breakpoint Cluster Region - Abelson Gene; BIHAM: Institute of Primary Health Care of Bern; BM: Bone Marrow; BP: Blast Phase; CCyR: Complete Cytogenetic Response; CINeMA: Confidence in Network Estimates; CML: Chronic Myeloid Leukemia; CP: Chronic Phase; EHA: European Haematology Association; EMR: Early Molecular Response; ESMO: European Society for Medical Oncology; FISH: Fluorescent in Situ Hybridization; GRADE: Grading of Recommendations Assessment, Development and Evaluation; IRCCS: Istituto di Ricovero e Cura a Carattere Scientifico; MMR: Major Molecular Response; MR: Molecular Response; NCCN: National Comprehensive Cancer Network; NMA: Network Meta-Analysis; PFS: Progression-Free Survival; Ph+: Philadelphia Chromosome-Positive; PROSPERO: International Prospective Register of Systematic Reviews; RCT: Randomised Controlled Trial; RoB: Risk of Bias; RT-PCR: Reverse Transcription Polymerase Chain Reaction; OS: Overall Survival; SIE: Italian Society of Hematology; SUCRA: Cumulative Ranking Curve; TKI: Tyrosine Kinase Inhibitor.

\section{Declarations}

\section{Ethics approval and consent to participate}

Not applicable.

\section{Consent for publication}

Not applicable.

\section{Availability of data and materials}

Databases created are available for purposes of data sharing.

\section{Competing interests}

The authors declare that they have no conflict interest.

\section{Funding}

Not applicable.

\section{Authors' contributions}

C.V., B.F., designed and wrote the review protocol; F.A., G.S., G.P., D.K.H. conceived the review protocol; C.D.G. conceived, led and coordinated the entire protocol process. All authors were responsible for revising the manuscript critically for important intellectual contents. All authors read and approved the final manuscript. C.V. and C.D.G. had the final responsibility to submit for publication.

\section{References}


1 Faderl S, Talpaz M, Estrov Z, O'Brien S, Kurzrock R, Kantarjian HM. The biology of chronic myeloid leukemia. N Engl J Med. 1999;341:164-72.

2 Sant M, Allemani C, Tereanu C, De Angelis R, Capocaccia R, Visser O, et al. Incidence of hematologic malignancies in Europe by morphologic subtype: results of the HAEMACARE project. Blood. 2010;116(19):3724-34.

3 Hoffmann VS, Baccarani M, Hasford J, Lindoerfer D, Burgstaller S, Sertic D, et al. The EUTOS population-based registry: incidence and clinical characteristics of $2904 \mathrm{CML}$ patients in 20 European Countries. Leukemia. 2015;29(6):1336-43.

4 Druker BJ, Talpaz M, Resta DJ, Peng B, Buchdunger E, Ford JM, et al. Efficacy and safety of a specific inhibitor of the BCR-ABL tyrosine kinase in chronic myeloid leukemia. N Engl J Med. 2001;344(14):10317.

5 https://ec.europa.eu/health/documents/communityregister/2017/20170508137859/anx_137859_it.pdf (accessed 01 October 2020).

6 Radich JP, Kopecky KJ, Appelbaum FR, Kamel-Reid S, Stock W, Malnassy G, et al. A randomized trial of dasatinib 100 mg versus imatinib 400 mg in newly diagnosed chronic-phase chronic myeloid leukemia. Blood. 2012;120(19):3898-905.

7 Kantarjian HM, Shah NP, Hochhaus A, Cortes J, Shah S, Ayala M, et al. Dasatinib versus imatinib in newly diagnosed chronic-phase chronic myeloid leukemia. N Engl J Med. 2010;362(24):2260-70.

8 Hjorth-Hansen H, Stenke L, Söderlund S, Dreimane A, Ehrencrona H, Gedde-Dahl T, et al. Dasatinib induces fast and deep responses in newly diagnosed chronic myeloid leukaemia patients in chronic phase: clinical results from a randomised phase-2 study (NordCML006). Eur J Haematol. 2015;94(3):24350 .

9 Saglio G, Kim DW, Issaragrisil S, le Coutre P, Etienne G, Lobo C, et al. Nilotinib versus imatinib for newly diagnosed chronic myeloid leukemia. N Engl J Med. 2010;362(24):2251-9.

10 http://www.ema.europa.eu/docs/it_IT/document_library/EPAR_-

_Product_Information/human/000709/WC500056998.pdf (accessed 01 October 2020).

11 https://ec.europa.eu/health/documents/communityregister/2017/20170524137917/anx_137917_it.pdf (accessed 01 October 2020).

12 https://ec.europa.eu/health/documents/communityregister/2013/20130327125474/anx_125474_it.pdf (accessed 01 October 2020).

13 Cortes JE, Kim DW, Kantarjian HM, Brümmendorf TH, Dyagil I, Griskevicius L, et al. Bosutinib versus imatinib in newly diagnosed chronic-phase chronic myeloid leukemia: results from the BELA trial. J Clin 
Oncol. 2012;30(28):3486-92.

14 Cortes JE, Gambacorti-Passerini C, Deininger MW, Mauro MJ, Chuah C, Kim DW, et al. Bosutinib Versus Imatinib for Newly Diagnosed Chronic Myeloid Leukemia: Results From the Randomized BFORE Trial. J Clin Oncol. 2018;36(3):231-7.

15 https://ec.europa.eu/health/documents/communityregister/2016/20160825135744/anx_135744_it.pdf (accessed 01 October 2020).

16 Lipton JH, Chuah C, Guerci-Bresler A, Rosti G, Simpson D, Assouline S, et al. Ponatinib versus imatinib for newly diagnosed chronic myeloid leukaemia: an international, randomised, open-label, phase 3 trial. Lancet Oncol. 2016;17(5):612-21.

17 Hochhaus A, Larson RA, Guilhot F, Radich JP, Branford S, Hughes TP, et al. Long-Term Outcomes of Imatinib Treatment for Chronic Myeloid Leukemia. N Engl J Med. 2017;376(10):917-27.

18 Osca-Gelis G, Puig-Vives M, Saez M, Gallardo D, Lloveras N, Guàrdia R, et al. Is survival in myeloid malignancies really improving? A retrospective 15 year population-based study. Leuk Lymphoma. 2015;56(4):896-902.

19 Sant M, Minicozzi P, Mounier M, Anderson LA, Brenner H, Holleczek B, et al. Survival for haematological malignancies in Europe between 1997 and 2008 by region and age: results of EUROCARE-5, a population-based study. Lancet Oncol. 2014;15(9):931-42.

20 Wiggins CL, Harlan LC, Nelson HE, Stevens JL, Willman CL, Libby EN, et al. Age disparity in the dissemination of imatinib for treating chronic myeloid leukemia. Am J Med. 2010;123(8):764.e1-9.

21 Hochhaus A, Saussele S, Rosti G, Mahon FX, Janssen JJWM, Hjorth-Hansen H, et al; ESMO Guidelines Committee. Chronic myeloid leukaemia: ESMO Clinical Practice Guidelines for diagnosis, treatment and follow-up. Ann Oncol. 2017;28 (Supplement 4): iv41-iv51.

22 Radich JP, Deininger M, Abboud CN, Altman JK, Berman E, Bhatia R, et al. Chronic Myeloid Leukemia, Version 1.2019, NCCN Clinical Practice Guidelines in Oncology. J Natl Compr Canc Netw. 2018;16(9):1108-35.

23 Vener C, Banzi R, Ambrogi F, Ferrero A, Saglio G, Pravettoni G, et al. First-line imatinib vs second- and third-generation TKIs for chronic phase CML: a systematic review and meta-analysis. Blood Advances. 2020;4(12):2723-35. doi: 10.1182/bloodadvances.2019001329.

24 http://methods.cochrane.org/bias/assessing-risk-bias-included-studies (accessed 01 October 2020). 25 http://gradepro.org/ (accessed 01 October 2020). 
26 Cipriani A, Higgins JP, Geddes JR, Salanti G. Conceptual and technical challenges in network metaanalysis. Ann Intern Med. 2013;159:130-37.

27 http://www.crd.york.ac.uk/PROSPERO/ (accessed 01 October 2020).

28 https://cinema.ispm.unibe.ch/ (accessed 01 October 2020).

29 Higgins JP, Thompson SG, Deeks JJ, Altman DG. Measuring inconsistency in meta-analyses. BMJ. 2003; 327:557-60.

30 Veroniki AA, Vasiliadis HS, Higgins JP, Salanti G. Evaluation of inconsistency in networks of interventions. Int J Epidemiol. 2013;42:332-45.

31 Higgins JP, Jackson D, Barrett JK, Lu G, Ades AE, White IR. Consistency and inconsistency in network meta-analysis: concepts and models for multi-arm studies. Res Synth Methods. 2012;3:98-110.

32 Salanti G, Ades AE, loannidis JP. Graphical methods and numerical summaries for presenting results from multiple-treatment meta-analysis: an overview and tutorial. J Clin Epidemiol. 2011;64(2):163-71.

33 Nikolakopoulou A, Mavridis D, Furukawa TA, Cipriani A, Tricco AC, Straus SE, et al. Living network meta-analysis compared with pairwise meta-analysis in comparative effectiveness research: empirical study. BMJ. 2018;360:k585.

\section{Supplementary Files}

This is a list of supplementary files associated with this preprint. Click to download.

- Appendix.Searchstrategy27102020.docx

- PRISMAP2015checklist27102020.docx 